\title{
Pleofungins, Novel Inositol Phosphorylceramide Synthase Inhibitors, from Phoma sp. SANK 13899
}

\author{
I. Taxonomy, Fermentation, Isolation, and Biological Activities \\ Tatsuya Yano, Azusa Aoyagi, Shiho Kozuma, Yoko Kawamura, Isshin Tanaka, \\ Yasuhiro Suzuki, Yasuyuki Takamatsu, Toshio Takatsu, Masatoshi Inukai
}

Received: October 26, 2006 / Accepted: January 29, 2007

(C) Japan Antibiotics Research Association

\begin{abstract}
In the course of a screening for inositol phosphorylceramide (IPC) synthase inhibitors, the novel inhibitors pleofungins $\mathrm{A}, \mathrm{B}, \mathrm{C}$, and $\mathrm{D}$ were found in a mycelial extract of a fungus, Phoma sp. SANK13899. Purification was performed by $50 \%$ methanol and ethyl acetate extraction, reversed phase open-column chromatography, and HPLC separations. Pleofungin A inhibited the IPC synthase of Saccharomyces cerevisiae and Aspergillus fumigatus at $\mathrm{IC}_{50}$ values of 16 and $1.0 \mathrm{ng} / \mathrm{ml}$, respectively. The inhibitor also suppressed the growth of Candida albicans, Cryptococcus neoformans, and A. fumigatus at MIC values of 2.0, 0.3 , and $0.5 \mu \mathrm{g} / \mathrm{ml}$, respectively. These biological properties indicate that pleofungins belong to a novel class of IPC synthase inhibitors efficacious against $A$. fumigatus.
\end{abstract}

Keywords sphingolipid, inositol phosphoryceramide synthase, anti-fungal, Aspergillus fumigatus, pleofungin

T. Yano (Corresponding author), S. Kozuma, Y. Kawamura, M. Inukai: Lead Discovery Research Laboratories, Sankyo Co., Ltd., 1-2-58 Hiromachi, Shinagawa-ku, Tokyo 140-8710, Japan, E-mail: tyyano@sankyo.co.jp

A. Aoyagi, I. Tanaka, T. Takatsu: Core Technology Research Laboratories, Sankyo Co., Ltd., 1-2-58 Hiromachi, Shinagawa-ku, Tokyo 140-8710, Japan

Y. Suzuki, Y. Takamatsu: Process Development Laboratories, Sankyo Co., Ltd., 389-4 Shimokawa Otsurugi, Izumimachi, Iwaki-shi, Fukushima 971-8183, Japan

\section{Introduction}

Incidence of systemic fungal disease has increased during the last two decades and now fungal infections are recognized as one of the major life threatening diseases. However, the options of clinically available antifungal drugs are limited, and these drugs also have problems in their severe side effects and emergence of resistance in several pathogens. Fungicidal agents effective to Aspergillus fumigatus are of particular interest because of the rapid progression of this infection which is difficult to control, and generally fatal.

Inositol phosphorylceramide (IPC) synthase is recently reported as one of the novel targets for antifungal drugs because these lipids are not detectable in mammalian cells, in which sphingomyelin seems to be the counterpart of IPC [1]. IPC and its derivatives are the major species of sphingolipids and share $30 \%$ of the total phospholipids [2, $3]$ in yeast, and these sphingolipids and their precursors are known to regulate cell growth and death in the Aspergillus species [4] as well as in yeast [5 9]. Aureobasidin A [10], rustmicin [11, 12], and khafrefungin [13] have already been reported to inhibit IPC synthase potently, and also to suppress the growth of Saccharomyces cerevisiae, Candida albicans, and Cryptococcus neoformans. All these known IPC synthase inhibitors, however, lack fungicidal activity against $A$. fumigatus.

During the screening of IPC synthase inhibitors with an anti-A. fumigatus effect, we isolated pleofungins $\mathrm{A}, \mathrm{B}, \mathrm{C}$, and D in the mycelial extract of a fungus Phoma sp. SANK13899. In this paper, we report the taxonomy and fermentation of the producing fungus, and also isolation and biological properties of pleofungins. The structure 
elucidation of them is published in the following paper in this issue [22].

\section{Materials and Methods}

\section{Taxonomic Studies}

The producing fungus SANK13899 was isolated from a soil sample collected at Tokyo, Japan. For identification of the fungus, the following media were used; potato dextrose agar (PDA, Nissui, Tokyo), corn meal agar (CMA, Nissui, Tokyo) and Miura's medium (LCA) [14]. The observation of the colonies was made after a two-week incubation at $23^{\circ} \mathrm{C}$. The color names used in this study were taken from Kornerup and Wanscher [15].

\section{Fermentation and Purification}

In order to acquire the pleofungins $\mathrm{A}$ and $\mathrm{B}$, the strain Phoma sp. SANK13899 was inoculated from a slant culture into $500 \mathrm{ml}$ of sterilized seed cultivation medium consisting of glycerol $3.0 \%$, glucose $3.0 \%$, soluble starch $2.0 \%$, soybean meal $1.0 \%$, gelatin $0.25 \%$, yeast extract $0.25 \%$, $\mathrm{NH}_{4} \mathrm{NO}_{3} \quad 0.25 \%$ and cultured in a 2-liter Erlenmeyer flask by shaking on a rotary shaker $(210 \mathrm{rpm})$ at $23^{\circ} \mathrm{C}$ for 6 days. The culture (1.5 liters) was transferred to 30 liters of the fresh seed medium in a 60-liter tank-fermentor, and cultured at $23^{\circ} \mathrm{C}$ for 2 days. Then, 15 liters of the preculture was transferred to 300 liters of fermentation medium-1 consisting of glucose $8.0 \%$, malt extract $2.0 \%$, yeast extract $0.2 \%$, tripton $1.0 \%, \mathrm{NH}_{4} \mathrm{NO}_{3} 0.1 \%, \mathrm{NaNO}_{3}$ $0.1 \%, \mathrm{KH}_{2} \mathrm{PO}_{4} 0.1 \%, \mathrm{MgSO}_{4} \cdot 7 \mathrm{H}_{2} \mathrm{O} 0.1 \%$ in a 600 -liter tank-fermentor and fermented at $23^{\circ} \mathrm{C}$ for 7 days. For the production of pleofungins $\mathrm{C}$ and $\mathrm{D}$, the strain was precultured in the same way as described above and $750 \mathrm{ml}$ of the pre-culture was transferred into 15 liters of fermentation medium-2 consisting of glucose $10 \%$, malt extract 2.0\%, Pharmamedia 1.0\%, GE90M 1.0\%, $\mathrm{NH}_{4} \mathrm{NO}_{3}$ $0.1 \%, \mathrm{NaNO}_{3} 0.1 \%, \mathrm{KH}_{2} \mathrm{PO}_{4} 0.1 \%, \mathrm{MgSO}_{4} \cdot 7 \mathrm{H}_{2} \mathrm{O} 0.1 \%$ in a 30 -liter jar-fermentor and fermented at $23^{\circ} \mathrm{C}$ for 7 days.

In order to purify the active compounds from the culture broth, Celite 545, Cosmosil 140 C18 OPN, YMC Pack ODS-AM, and Shodex Asahipak C8P 90 2F were purchased from Celite Corporation, NACALAI TESQUE, INC., YMC Co., Ltd., and Showa Denko K.K., respectively.

\section{Analysis of IPC Synthase Activity}

Crude IPC synthase of $A$. fumigatus was prepared as follows. The spores of $A$. fumigatus IAM $2034\left(3.0 \times 10^{8}\right.$ cells) were inoculated in $100 \mathrm{ml}$ of $0.2 \%$ agar containing YPD medium (yeast extract $10 \%$, polypeptone $20 \%$, glucose $20 \%$ ), and cultured at $26^{\circ} \mathrm{C}$ for 2 days to acquire stationary growing fungal cells. The culture $(10 \mathrm{ml})$ was transferred to $100 \mathrm{ml}$ of fresh YPD medium and further cultured at $26^{\circ} \mathrm{C}$ for 16 hours to bring the cells to an exponentially growing phase. The cells were harvested by a filtration with a filter paper (Whatman) and washed once with ice-cold lysis buffer $(50 \mathrm{mM}$ potassium phosphate $\mathrm{pH}$ 6.5, $10 \mathrm{mM}$ EDTA, $5 \mathrm{mM}$ dithiothreitol, $1 \mathrm{mM}$ PMSF, $10 \mu \mathrm{g} / \mathrm{ml}$ leupeptin, $10 \mu \mathrm{g} / \mathrm{ml}$ pepstatin A). The wet mycelium $(50 \mathrm{~g})$ was resuspended in $400 \mathrm{ml}$ of ice-cold lysis buffer and $250 \mathrm{ml}$ of chilled glass beads (SIGMA), and stirred with Dyno-Mil 3 times at $2000 \mathrm{rpm}$ for 30 seconds with 1 minute intervals. The cell lysate was passed through cheesecloth and the flow-throughs were centrifuged for 1 hour at $10,000 \times g$ to remove cell debris and glass beads. The resultant supernatant was concentrated by an ultrafiltration with a YM-30 membrane (Millipore). Finally, the concentrated material was resuspended in the lysis buffer containing $20 \%$ of glycerol and stored at $-80^{\circ} \mathrm{C}$.

For preparation of crude IPC synthase of $S$. cerevisiae, $S$. cerevisiae AHP250 strain was cultured with 1 liter of YPD medium at $30^{\circ} \mathrm{C}$ to $\mathrm{OD}_{660}$ of 4.0 . The yeast cells were processed and their microsomes were acquired as previously described [16]. The microsomes were dissolved in the lysis buffer and stored at $-80^{\circ} \mathrm{C}$.

To measure the $A$. fumigatus IPC synthase activities in vitro, $30 \mu \mathrm{g}$ of crude enzyme was mixed with liposomes containing 1.6 $\mu \mathrm{M}$ BODIPY-FL-C5-ceramide (Molecular Probes) and $89 \mu \mathrm{M}$ phosphatidyl inositol (SIGMA) in $50 \mu \mathrm{l}$ of reaction buffer $(10 \mathrm{mM}$ potassium phosphate $\mathrm{pH} 6.5$, $2 \mathrm{mM}$ dithiothreitol, $1.4 \mathrm{mM}$ PMSF, $14 \mu \mathrm{g} / \mathrm{ml}$ leupeptin, $14 \mu \mathrm{g} / \mathrm{ml}$ pepstatin A), and incubated at $32^{\circ} \mathrm{C}$ for 4 hours. For evaluation of $S$. cerevisiae IPC synthase activity, $12.5 \mu \mathrm{g}$ of $S$. cerevisiae microsomes was mixed with the same liposomes as described above in $50 \mu \mathrm{l}$ of reaction buffer (10 mM Tris-HCl pH 7.5, $1 \mathrm{mM}$ EDTA) and incubated at $30^{\circ} \mathrm{C}$ for 1 hour. To test the inhibitory activities of the samples, the final $2 \%$ of the samples were included in the reactions. After the incubation of the reaction mixtures, BODIPY-labeled IPC was separated by the addition of solvent mixture $(\mathrm{MeOH}$ : diethylether, $41: 91$ ), and their fluorescent values were measured. The specific activities of the crude IPC synthase in the $A$. fumigatus cell lysate and $S$. cerevisiae microsome were 0.26 and 2.4 (nM of BODIPY-IPC formed $/ \mathrm{sec} / \mathrm{mg}$ of protein), and $\mathrm{Km}$ values for BODIPY-C5-ceramide were 5.9 and $5.2 \mu \mathrm{M}$, respectively. The reactions were over $50 \%$ complete based on TLC analysis of BODIPY-labeled IPC conversion product. 


\section{Analysis of de novo Sphingolipid Biosynthesis}

In order to assess the inhibitory effects of inhibitors on the biosynthesis of the sphingolipids in A. fumigatus, exponentially growing cells were prepared by a precultivation of the A. fumigatus spores $\left(2.5 \times 10^{7}\right.$ cells $)$ for 12 hours at $26^{\circ} \mathrm{C}$. The cells were further cultured with $5 \mu \mathrm{Ci} / \mathrm{ml}$ of ${ }^{3} \mathrm{H}$-dihydrosphingosine (ARC) and inhibitors for 6 hours at $30^{\circ} \mathrm{C}$. Cell pellets were acquired by centrifugation for 3 minutes at $3000 \mathrm{rpm}$, and washed 3 times with ice-cold water. The pellets were suspended in $1 \mathrm{ml}$ of solvent mixture (95\% ethanol: distilled water: diethylether: pyridine : $\left.\mathrm{NH}_{4} \mathrm{OH}, 15: 15: 5: 5: 0.018\right)$, and the total lipids were extracted by heating for 30 minutes at $60^{\circ} \mathrm{C}$ [17]. The lipid extracts were dried under an $\mathrm{N}_{2}$ gas flow and dissolved in $100 \mu \mathrm{l}$ of dichloromethane: methanol $(1: 1, \mathrm{v} / \mathrm{v})$. Total lipid solutions $(20 \mu \mathrm{l})$ were spotted on the silica-gel thin-layer chromatography plates $(20 \mathrm{~cm}$ Whatman LK5) and developed with dichrolomethane : methanol: $2 \mathrm{~N}$ acetic acid $(6: 4: 1, \mathrm{v} / \mathrm{v})$. Visualization and densitometric analysis of $\left[{ }^{3} \mathrm{H}\right]$-labeled lipids were performed with a BAS-2000 imaging analyzer (FUJI film).

For analysis of the IPC biosynthesis in S. cerevisiae, $1.0 \times 10^{7}$ cells $/ \mathrm{ml}$ of exponentially growing $S$. cerevisiae YPH250 cells were prepared in a synthetic complete medium $[0.67 \%$ Bacto-yeast nitrogen base without amino acids (Difco), 2.0\% glucose; supplemented with amino acids], and further cultured with $10 \mu \mathrm{Ci}$ of ${ }^{3} \mathrm{H}-$ dihydrosphingosine (ARC) and inhibitors for 1.5 hours at $30^{\circ} \mathrm{C}$. After the incubation, cell pellets were obtained by centrifugation at $3,000 \times g$ for 10 minutes and washed with $2 \mathrm{ml}$ of ice-cold water. The total lipids were extracted from the pellets as described above. The lipid extracts were dried and dissolved in the same solvent as described above. Aliquots of the solutions were spotted on the silica-gel thinlayer chromatography plates $(20 \mathrm{~cm}$ Whatman LK5) and developed with dichloromethane : methanol: $2 \mathrm{~N} \mathrm{NH}_{4} \mathrm{OH}$ $(9: 7: 2, \mathrm{v} / \mathrm{v})$. Visualizations and analyses were carried out in the same way as described in A. fumigatus $\left[{ }^{3} \mathrm{H}\right]$-labeled lipids. The respective sphingolipids were detected by comparing the TLC profiles of the wild type, $\operatorname{sur} 2 \Delta, \operatorname{csg} 2 \Delta$, and $\operatorname{ccc} 2 \Delta$ strain.

For analysis of sphingomyelin biosynthesis, $1.0 \times 10^{6}$ cells of the A549 human lung carcinoma cell line were preincubated with inhibitors for 1 hour and further incubated for 24 hours after the addition of $10 \mu \mathrm{Ci}$ of $\left[{ }^{3} \mathrm{H}\right]$ choline (NEN). Cells were scraped with a rubber policeman and washed once with ice cold PBS. The total lipids were extracted from the cell pellets as described above. Aliquots of the total lipids were developed on the silica-gel thin-layer chromatography with a solvent system, dichloromethane: methanol: $2 \mathrm{mM} \mathrm{MgCl}_{2}(65: 25: 4, \mathrm{v} / \mathrm{v})$, and analyzed as described above.

\section{Antifungal Activities}

Antifungal activities were measured following the National Committee for Clinical Laboratory Standard method [18]. For determination of MIC against Candida species and Cryptococcus neoformans, cells were diluted into $5.0 \times 10^{3} \mathrm{CFU} / \mathrm{ml}$ in RPMI medium. The cell suspensions $(100 \mu \mathrm{l})$ were transfered onto $96-$-well microtiter plates. And then, $100 \mu$ of diluted samples with RPMI medium were added to the cell suspensions on the microtiter plates. After the mixing of the samples and cell suspensions of Candida and Cryptococcus, the plates were incubated at $37^{\circ} \mathrm{C}$ for 24 and 48 hours, respectively. The growth conditions were determined by naked-eye observation by measuring the $\mathrm{OD}_{600}$. For MICs against the Aspergillus species, the spore number was adjusted to $3.0 \times 10^{4} \mathrm{CFU} / \mathrm{ml}$ in RPMI medium. Cell suspensions $(90 \mu \mathrm{l})$ were distributed onto $96-$ well microtiter plates and further added with $100 \mu$ of diluted samples and $10 \mu \mathrm{l}$ of Alamour Blue. Then, the plates were incubated at $26^{\circ} \mathrm{C}$ for 72 hours and the growth conditions were determined by measuring the $\mathrm{OD}_{595}$.

For evaluation of minimal fungicidal concentrations (MFCs) against Candida, Cryptococcus, and Aspergillus, $1.5 \mu \mathrm{l}$ of the mixtures were pipetted from 96-well microtiter plates after the MIC measurements and spread on SDA (Sabouraud Dextrose Agar) or PDA (Potato Dextrose Agar) plates. Then, the number of colonies was counted. The concentrations at which the colony numbers were less than 4 or 20 were defined as the MFCs against Candida and Cryptococcus, or Aspergillus, respectively.

\section{Results}

\section{Taxonomy of Producing Organism}

Colonies on the PDA plates reached $13 \sim 17 \mathrm{~mm}$ in diameter. The colony surface was tomentose, floccus at the center and colored Grey (3B1) to White. The reverse side of the colonies was Brown (6E5 4). Colonies on the CMA plates reached $15 \sim 17 \mathrm{~mm}$ in diameter. The colony surface was farinaceous and colored Greyish Green (27E5) to Dull Green (27E4). The reverse side of the colonies was Dark Green (27F4). Formation of pycnidia was observed after a one-month incubation on LCA. The pycnidia were $100 \sim 200 \mu \mathrm{m}$ in diameter, globose to subglobose, immersed to subimmersed in the medium and colored brown to black (Fig. 1A). The conidiogenous cells were $7.5 \sim 9 \times 1.5 \sim 2 \mu \mathrm{m}$ in size, phialidic, cylindrical to ampulliform and hyaline. The conidia were 


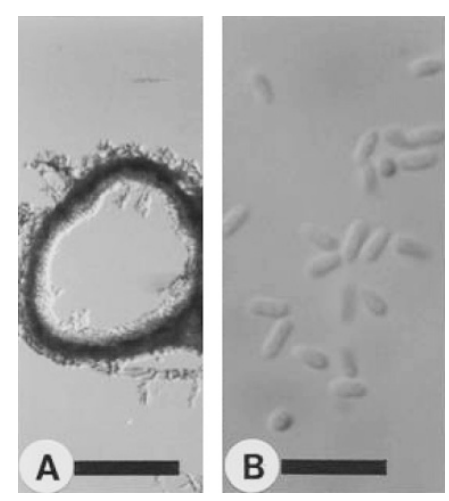

Fig. 1 Morphology of the strain SANK 13899.

A) Section of the pycnidium. Bar represents $100 \mu \mathrm{m}$. B) Conidia. Bar represents $10 \mu \mathrm{m}$.

$3.5 \sim 5 \times 1.5 \sim 2 \mu \mathrm{m}$ in size, cylindrical, one-celled and hyaline (Fig. 1B).

The above characteristics of the strain SANK13899 indicate that this fungus belongs to the genus Phoma [19]. The strain has been deposited at the National Institute of Bioscience and Technology, Japan as Phoma sp. SANK13899 under the accession number of FERM BP6851. In addition, this strain was clustered within the Pleosporales by means of the molecular phylogenetic analysis based on the 18S rDNA gene sequence (data not shown).

\section{Isolation of Pleofungins A, B, C and D}

Fermentation of SANK13899 strain was carried out as in described in materials and methods. The harvested broth (370 liters) was added with $18 \mathrm{~g}$ of Diatomaceous Earth (Celite 545) and which was then filtered to acquire supernatant and mycelium. The active material was extracted from the mycelium by adding 50\% methanol $(\mathrm{MeOH})$ followed by $\mathrm{pH}$ adjustment ( $\mathrm{pH} 2.0$ ) and filtration. A 310-liter aliquot of the filtrate was applied onto 30 liters of a reversed phase open column (Cosmosil $140 \mathrm{C} 18$ OPN) which was equilibrated with $50 \% \mathrm{MeOH}$ containing $0.05 \%$ of trifluoroacetic acid (TFA). After washing the column with 270 liters of $50 \% \mathrm{MeOH}$ containing $0.05 \%$ TFA and 100 liters of $40 \%$ acetonitrile $(\mathrm{MeCN})$ containing $0.05 \%$ TFA, the active compounds were eluted with $60 \% \mathrm{MeCN}$ containing $0.05 \%$ TFA and the active fractions were pooled (30 liters). Active compounds were further extracted from the pooled fractions with 50 liters of ethyl acetate (EtOAc) after $\mathrm{pH}$ neutralization with $\mathrm{NaOH}$. The extract was washed with saturated saline, dried over with anhydrous $\mathrm{Na}_{2} \mathrm{SO}_{4}$ and concentrated in vacuo to obtain $32.3 \mathrm{~g}$ of crude oily material. Further purification was performed by a primary HPLC (column, YMC Pack ODS-AM, 100 i.d. $\times 500$ mm; mobile phase, $\mathrm{MeCN}: 1.0 \%$ triethylamine-phosphate buffer (pH 6.0), 3:1; flow rate, $240 \mathrm{ml} /$ minute; detection, UV at $210 \mathrm{~nm}$ ). The eluate (26 liters) containing pleofungins A and $\mathrm{B}$ was mixed with 20 liters of water and EtOAc $(1: 1)$ to extract the active compounds. The EtOAc phase was washed, dried over and concentrated as described above to yield $7.8 \mathrm{~g}$ of oily material. A $326 \mathrm{mg}$ aliquot of the material was dissolved in $\mathrm{MeOH}$, and subjected to repeated secondary HPLC (column, Shodex Asahipak C8P 90 2F, 20 i.d. $\times 250 \mathrm{~mm}$; mobile phase, $60 \% \mathrm{MeCN}$ containing $10 \mathrm{mM} \mathrm{NH}_{4} \mathrm{HCO}_{3}$; flow rate, $14 \mathrm{ml} /$ minute; detection, UV at $210 \mathrm{~nm}$ ). HPLC fractions possessing the aimed activity were combined and concentrated to acquire pure pleofungin A as a colorless powder $(275 \mathrm{mg})$. In order to prepare pleofungin $\mathrm{B}$, a $2.1 \mathrm{~g}$ aliquot of the oily material prepared by primary HPLC as mentioned above was dissolved in $\mathrm{MeOH}$ and applied onto $170 \mathrm{ml}$ of a reversed phase open column (resin, Cosmosil 140 C18 OPN; mobile phase, 60 to $90 \% \mathrm{MeCN}$ containing $0.05 \%$ TFA, linear gradient). Active fractions were pooled and concentrated by evaporation and lyophilization to give pale yellow powder $(109 \mathrm{mg})$. Finally, the powder was purified by HPLC (column, Shodex Asahipak C8P 90 2F, 20 i.d. $\times 250 \mathrm{~mm}$;

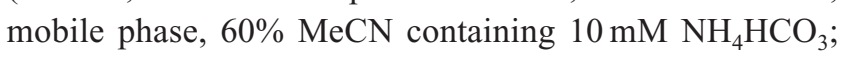
flow rate, $14 \mathrm{ml} /$ minute; detection, $\mathrm{UV}$ at $210 \mathrm{~nm}$ ). Pure pleofungin B $(69.5 \mathrm{mg})$ was acquired as a colorless powder after concentration of the active fractions.

For purification of pleofungins $\mathrm{C}$ and $\mathrm{D}$, the harvested broth (55 liters) was processed in the same manner as described above, and the mycelium was subjected to $50 \%$ $\mathrm{MeOH}$ extraction. After the filtration and $\mathrm{pH}$ neutralization of the extract, the active compounds were further extracted with EtOAc. Oily material $(107 \mathrm{mg})$ was obtained from the EtOAc phase by washing and concentration as described above. This material was dissolved in $\mathrm{MeOH}$ and applied to a reversed phase open column (resin, 3 liters of Cosmosil $140 \mathrm{C} 18$ OPN; mobile phase, $30 \% \mathrm{MeCN}$ containing $0.05 \% \mathrm{TFA}$ ), and consequently $3.8 \mathrm{~g}$ of oily material was acquired from the combined active fractions. This material was further purified by repeating the preparative HPLC (column, Shodex Asahipak C8P 90 2F, 20 i.d. $\times 250 \mathrm{~mm}$; mobile phase, $60 \% \mathrm{MeCN}$ containing $10 \mathrm{mM} \mathrm{NH}_{4} \mathrm{HCO}_{3}$; flow rate, $14 \mathrm{ml} /$ minute; detection, UV at $210 \mathrm{~nm})$. Finally, pure pleofungins $\mathrm{C}(19.5 \mathrm{mg})$ and $\mathrm{D}(6.3 \mathrm{mg})$ were acquired as colorless powders after integration and concentration of the active fractions.

\section{Biological Properties of Pleofungin A}

Inhibitory activities of pleofungin A on IPC synthase of $A$. fumigatus and $S$. cerevisiae were evaluated by comparing them to those of aureobasidin A. As shown in Fig. 2A and 
A)

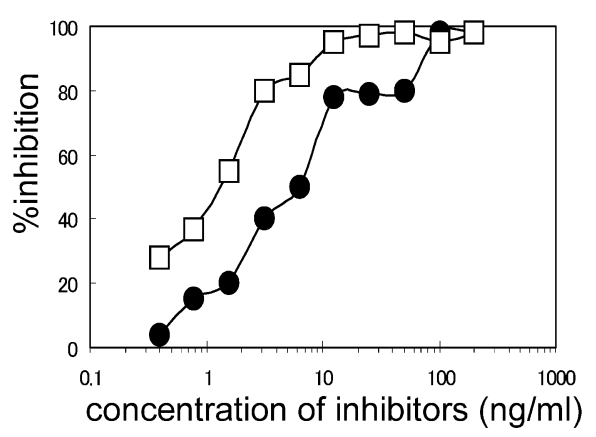

B)

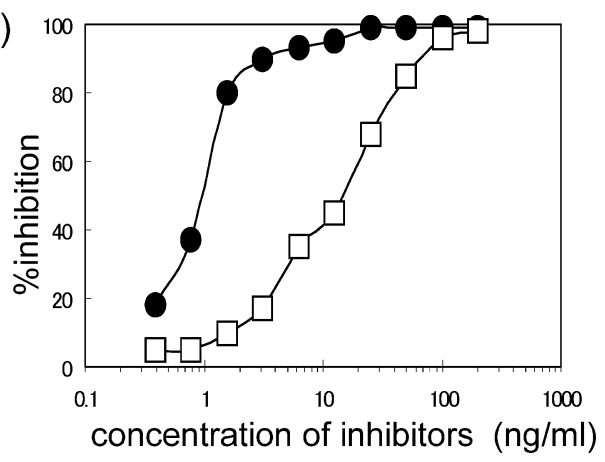

Fig. 2 Inhibition of IPC synthases by pleofungin A.

A) Inhibition of Saccharomyces cerevisiae IPC synthase, and B) Aspergillus fumigatus IPC synthase by pleofungin A. Closed circles and open squares represent the inhibitory activities of pleofungin A and aureobasidin A, respectively.

$\mathrm{B}$, pleofungin A strongly inhibited the A. fumigatus IPC synthase at an $\mathrm{IC}_{50}$ of $1.0 \mathrm{ng} / \mathrm{ml}$, while aureobasidin A inhibited the enzyme at an $\mathrm{IC}_{50}$ of $16 \mathrm{ng} / \mathrm{ml}$. However, pleofungin A was less effective against $S$. cerevisiae enzyme than aureobasidin $\mathrm{A}\left(\mathrm{IC}_{50}\right.$ of pleofungin $\mathrm{A}$ and aureobasidin A, 7.0 and $1.5 \mathrm{ng} / \mathrm{ml}$, respectively). IPC synthase inhibitory effects and the anti-fungal activities of pleofungins $\mathrm{B}, \mathrm{C}$ and $\mathrm{D}$ were much weaker than those of pleofungin $\mathrm{A}\left(\mathrm{IC}_{50}\right.$ of $\mathrm{B}, \mathrm{C}$, and $\mathrm{D}$ against $S$. cerevisiae IPC synthase, 80, 300, and $300 \mathrm{ng} / \mathrm{ml}$, respectively; MICs, Table 2). Thus, only pleofungin A was used for further analyses.

In the next stage, the effects of pleofungin A on de novo sphingolipid biosynthesis in A. fumigatus and S. cerevisiae were examined. As shown in Fig. 3A, pleofungin A completely suppressed the biosynthesis of $\left[{ }^{3} \mathrm{H}\right]$ dihydrosphingosine-derived lipids in A. fumigatus at the concentration of $0.5 \mu \mathrm{g} / \mathrm{ml}$, but aureobasidin A barely inhibited the synthesis of the same lipid even at $5.0 \mu \mathrm{g} / \mathrm{ml}$. In contrast, pleofungin A could not completely block the IPC biosynthesis in S. cerevisiae at less than $12 \mu \mathrm{g} / \mathrm{ml}$, whereas aureobasidin A inhibited it at $0.5 \mu \mathrm{g} / \mathrm{ml}$ (Fig. 3B). These patterns on the inhibition of the sphingolipid biosynthesis
A)

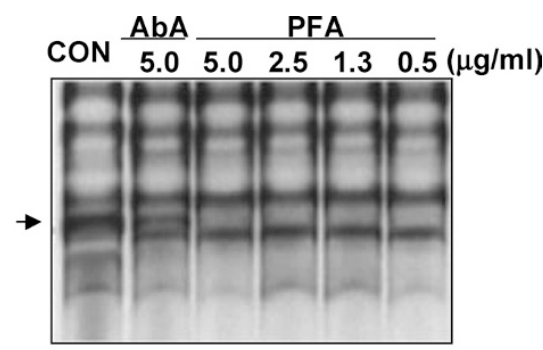

B)

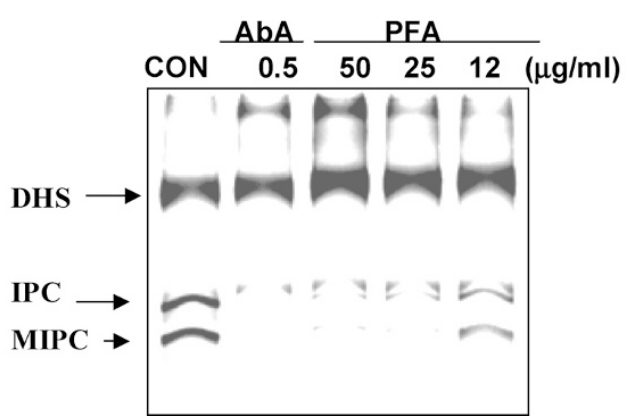

Fig. 3 Effects of pleofungin A on de novo sphingolipid biosynthesis.

A) Inhibition of dihydrosphingosine derived lipid biosynthesis in $A$. fumigatus by pleofungin $A$. An arrow indicates a dihydrosphingosine derived lipid whose biosynthesis is vulnerable to pleofungin $A$.

B) Inhibition of de novo IPC biosynthesis in S. cerevisiae by pleofungin A. S. cerevisiae were cultured with dihydrosphingosine and total lipids were analyzed as described in Materials and Methods. Abbreviations are as follows: PFA: pleofungin A, AbA: aureobasidin A, DHS: dihydrosphingosine, IPC: inositolphosphorylceramide, MIPC: mannosylinositolphosphorylceramide, CON: control (drug free).

by both inhibitors well reflected on their inhibitory activities against each enzyme. We next examined the effects of pleofungin A on the mammalian sphingolipid biosynthesis with using a culture cell line, A549, to confirm its selectivity for fungal enzymes over the corresponding mammalian counterpart. Pleofungin A showed no inhibition for de novo sphingomyelin biosynthesis in A549 cells at the concentrations up to $5.0 \mu \mathrm{g} / \mathrm{ml}$, demonstrating high specificity to the fungal sphingolipid biosynthesis (Fig. 4).

Antifungal properties of pleofungins against pathogenic fungi are shown as values of MIC and MFC, as well as those of the known IPC synthase inhibitors, aureobasidin A and rustmicin (Table 1). Pleofungin A showed growth inhibitory effects on the various kinds of fungi, but preferentially inhibited the growth of A. fumigatus and Cryptococcus neoformans. As previously reported, aureobasidin A and rustmicin effectively inhibited the growth of Candida species and Cryptococcus neoformans, but not that of $A$. fumigatus. Pleofungin A and aureobasidin 
Table 1 Antifungal spectrum of IPC synthase inhibitors

\begin{tabular}{|c|c|c|c|c|c|c|}
\hline & \multicolumn{2}{|c|}{ Pleofungin A } & \multicolumn{2}{|c|}{ Aureobasidin A } & \multicolumn{2}{|c|}{ Rustmicin } \\
\hline & MIC & MFC & MIC & MFC & MIC & MFC \\
\hline Candida albicans ATCC90028 & 2.0 & 5.0 & 0.5 & 0.6 & 2.5 & 10 \\
\hline C. parapsilosis IFO1396 & 0.5 & 2.5 & 0.5 & 1.3 & 0.6 & 0.6 \\
\hline C. grabrata ATCC90030 & 0.3 & 1.3 & 0.5 & 0.6 & 0.3 & 0.3 \\
\hline C. tropicalis IAM4185 & 2.0 & 2.5 & 0.5 & 0.6 & 1.3 & 0.3 \\
\hline Aspergillus fumigatus IAM2034 & 0.5 & 1.3 & $>20$ & $>20$ & $>20$ & $>20$ \\
\hline Cryptococcus neoformans IAM4772 & 0.3 & 0.3 & 1.0 & 1.3 & 0.3 & 0.3 \\
\hline Saccharomyces cerevisiae YPH250 & 10 & NT & 0.5 & NT & NT & NT \\
\hline
\end{tabular}

Minimum inhibitory concentration ( $\mathrm{MIC}, \mu \mathrm{g} / \mathrm{ml}$ ) and minimum fungicidal concentration (MFC, $\mu \mathrm{g} / \mathrm{ml}$ ) of inhibitors were measured as described in Materials and Methods. NT: not tested.

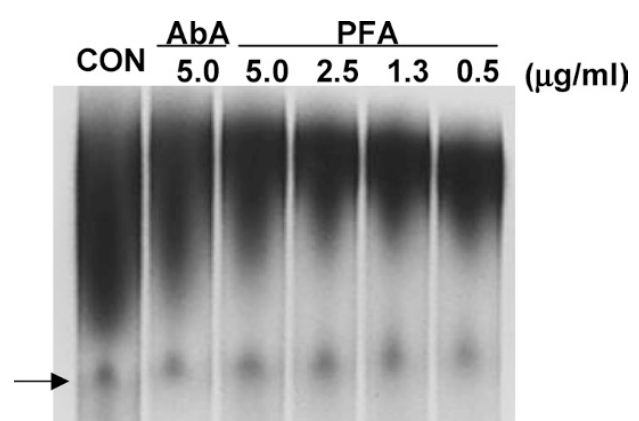

Fig. 4 Effect of pleofungin $A$ on sphingomyelin biosynthesis in A549 cells.

A549 cells were labeled with choline and their total lipids were analyzed in the presence of pleofungin $A$ and aureobasin A. An arrow indicates the choline labeled sphingomyelin. Abbreviations are as follows: PFA: pleofungin A, AbA: aureobasidin A, CON: control (drug free).

A also inhibited the growth of $S$. cerevisiae at MIC of 10 and $0.5 \mu \mathrm{g} / \mathrm{ml}$, respectively. These antifungal properties are consistent with their trends in their efficacies on IPC synthases and de novo sphingolipid biosynthesis. As in Table 1, almost all the MFC values of pleofungin A and the other inhibitors are close to the MIC values, suggesting that these IPC synthase inhibitors suppress the fungal growth through fungicidal effects.

\section{Discussion}

The novel IPC synthase inhibitors, pleofungins A, B, C, and D were isolated from the culture broth of Phoma sp. SANK13899. Pleofungin A more strongly inhibited IPC synthase of $A$. fumigatus than that of $S$. cerevisiae in our experimental conditions, however, aureobasidin A showed
Table 2 Antifungal spectrum of pleofungins (MIC, $\mu \mathrm{g} / \mathrm{ml}$ )

\begin{tabular}{lccc}
\hline & B & C & D \\
\hline Candida albicans ATCC90028 & $>65$ & 8.0 & 16 \\
C. parapsilosis IF01396 & $>64$ & 4.0 & 8.0 \\
C. grabrata ATCC90030 & 1.0 & 1.0 & 2.0 \\
C. tropicalis IAM4185 & $>64$ & 8.0 & $>64$ \\
Aspergillus fumigatus IAM2034 & $>64$ & 2.0 & 8.0 \\
Cryptococcus neoformans IAM4772 & 1.0 & 0.3 & 0.3 \\
\hline
\end{tabular}

an opposing tendency in the effects on those enzymes to pleofungin A. Pleofungin A also showed stronger growth inhibition against A. fumigatus and Cryptococcus neoformans than against Candida species and $S$. cerevisiae. It was previously indicated that the homology between Aspergillus and yeast IPC synthase genes is very low [20, 21], regardless of the high homology among the enzymes of Aspergillus species, such as A. fumigatus and $A$. nidulans. Our data suggested that pleofungin A may recognize the structural differences between the Aspergillus- and yeast-type enzymes, and preferably interact with Aspergillus-type enzymes. It was reported that the major cause of the aureobasidin A resistance of $A$. fumigatus is the increased efflux via an unknown transporter. However, even in the presence of an efflux inhibitor, such as verapamil, which sensitize A. fumigatus to a certain extent, the MIC of aureobasidin A against $A$. fumigatus is still higher than that against Candida albicans [22]. Those data suggest that the selectivity of the antifungal effect of aureobasidin A is also partly attributable to its potency of inhibition against the enzymes. It should be an intriguing subject to elucidate the determinants of the affinities of the inhibitors to $A$. 
fumigatus and S. cerevisiae IPC synthase, and generate more ideal inhibitors with a wider spectrum of antifungal effects than the known inhibitors.

It was also shown that pleofungin A has no inhibition against sphingomyelin biosynthesis in the A549 cell line, suggesting its selective action on fungal growth and low toxicity on animals. These data strongly support the possibility of the therapeutic effect of pleofungin A on $A$. fumigatus infected animals.

The lack of fungicidal action against $A$. fumigatus with previously reported inhibitors such as aureobasidin A, rustmicin, and khafrefungin, limits their potential for clinical development. Our discovery could facilitate the future research for the development of the IPC synthase inhibitors as a novel chemotherapeutic drug.

\section{References}

1. Nagiec MM, Nagiec EE, Baltisberger JA, Wells GB, Lester RL, Dickson RC. Sphingolipid synthesis as a target for antifungal drugs. Complementation of the inositol phosphorylceramide synthase defect in a mutant strain of Saccharomyces cerevisiae by the AURl gene. J Biol Chem 272: 9809-9817 (1997)

2. Dickson RC, Lester RL. Sphingolipid functions in Saccharomyces cerevisiae. Biochim Biophys Acta 1583: 13-15 (2002)

3. Daum G, Lees ND, Bard M, Dickson R. Biochemistry, cell biology and molecular biology of lipids of Saccharomyces cerevisae. Yeast 14: 1471-1510 (1998)

4. Cheng J, Park TS, Fischl AS, Ye XS. Cell cycle progression and cell polarity require sphingolipid biosynthesis in Aspergillus nidurans. Mol Cell Biol 21: 6198-6209 (2001)

5. Dickson RC, Nagiec EE, Skrzypek M, Tillman P, Wells GB, Lester RL. Sphingolipids are potential heat stress signals in Saccharomyces cerevisiae. J Biol Chem 272: 30196-31200 (1997)

6. Kim S, Fyrst H, Saba J. Accumulation of phosphorylated sphingoid long chain bases results in cell growth inhibition in Saccharomyces cerevisae. Genetics 156: 1519-1529 (2000)

7. Zhang X, Skrzypek MS, Lester RL, Dickson RC. Elevation of endogenous sphingolpid long-chain base phosphates kills Saccharomyces cerevisiae cells. Curr Genet 40: 221-233 (2001)

8. Jenkins GM, Hannun YA. Role for de novo sphingoid base biosynthesis in the heat-induced transient cell cycle arrest of Saccharomyces cerevisiae. J Biol Chem 276: 8574-8581 (2001)
9. Nickels JT, Broach JR. A ceramide-activated protein phosphatase mediates ceramide-induced G1 arrest of Saccharomyces cerevisae. Genes Dev 10: 382-394 (1996)

10. Takesako K, Kuroda H, Inoue T, Haruna F, Yoshikawa Y, Kato I, Uchida K, Hiratani T, Yamaguchi H. Biological properties of aureobasidin A, a cyclic depsipeptide antifungal antibiotic. J Antibiot 46: 1414-1420 (1993)

11. Mandala SM, Thornton RA, Milligan J, Rosenbach M, Garcia-Calvo M, Bull HG, Harris G, Abruzzo GK, Flattery AM, Gill CJ, Bartizal K, Dreikorn S, Kurtz MB. Rustmicin, a potent antifungal agent, inhibits sphingolipid synthesis at inositol phosphoceramide synthase. J Biol Chem 273: 14942-14949 (1998)

12. Takatsu T, Nakayama H, Shimazu A, Furihata K, Ikeda K, Furihata K, Seto H, Otake N. Rustmicin, a new macrolide antibiotic active against wheat stem rust fungus. J Antibiot 38: 1806-1809 (1985)

13. Mandala SM, Thornton RA, Rosenbach M, Milligan J, Garcia-Calvo M, Bull HG, Kurtz MB. Khafrefungin, a novel inhibitor of sphingolipid synthesis. J Biol Chem 272: 32709-32714 (1997)

14. Miura K, Kudo M. An agar-medium for aquatic Hyphomycetes. Trans Mycol Soc Japan 11: 116-118 (1970)

15. Kornerup A, Wansher JH. Methuen handbook of colour, 3rd ed. Methuen, London (1978)

16. Yano $\mathrm{T}$, Inukai M, Isono F. Deletion of OSH3 gene confers resistance against ISP-1 in Saccharomyces cerevisiae. Biochem Biophys Res Commun 27: 228-234 (2004)

17. Hanson BA, Lester RL. The extraction of inositol-containing phospholipids and phosphatidylcholine from Saccharomyces cerevisae and Neurospora crassa. J Lipid Res 21: 309-315 (1980)

18. Barchiesi F, Colombo AL, McGough DA, Rinaldi MG. Comparative study of broth macrodilution and microdilution techniques for in vitro antifungal susceptibility testing of yeasts by using the National Committee for Clinical Laboratory Standards' proposed standard. J Clin Microbiol 32: 2494-2500 (1994)

19. Sutton BC. The coelomycetes, fungi imperfecti with pycnidia, acervuli and stromata CMI, Kew (1980)

20. Kuroda M, Hashida-Okado T, Yasumoto R, Gomi K, Kato I, Takesako K. An aureobasidin A resistant gene isolated from Aspergillus is a homolog of yeast AUR1, a gene responsible for inositol phosphorylceramide (IPC) synthase activity. Mol Gen Genet 261: 290-296 (1999)

21. Heidler SA, Radding JA. Inositol phosphoryl transferase from pathogenic fungi. Biochem Biophys Acta 1500: 147-152 (2000)

22. Zhong W, Jeffries MW, Georgopapadakou NH. Inhibition of inositol phosphorylceramide synthase by aureobasidin A in Candida and Aspergillus species Antimicrob Agents Chemother 44: 651-653 (2000) 